\title{
Reflex sympatho-vagal coactivation and concealed QTc prolongation: Lessons from hERG-blockers and long QT syndromes
} type 1 and type 2

\author{
Pascal Champéroux ${ }^{1}$, Raafat Fares ${ }^{1}$, Sebastien Jude ${ }^{1}$, Vincent Maleysson ${ }^{1}$, Serge Richard ${ }^{1}$, \\ Jean-yves Le Guennec ${ }^{2}$, and Jerôme Thireau ${ }^{2}$ \\ ${ }^{1} \mathrm{ERBC}$ \\ ${ }^{2}$ INSERM U1046
}

February 20, 2021

\begin{abstract}
Background and Purposes: Several hERG blocking molecules known for their propensity in triggering Torsades de Pointes (TdP) were reported as increasing High Frequency QT oscillations (HFQT). This effect was found as reflecting a sympatho-vagal coactivation. The present work aims to characterise the mechanism(s) leading to this particular state of the autonomic nervous system. Experimental approach: Effects of 20 hERG blockers including 15 torsadogenic molecules were assessed by telemetry in beagle dogs. Electrocardiogram and stroke volume modelled from the pulse contour method were analysed at the first dose level causing either QTc prolongation and/or HFQT increase. Cardiac autonomic control was analysed using the High Frequency Autonomic Modulation (HFAM) model in dogs and in untreated genotyped LQT1 and LQT2 individuals, for comparison. Key results: The sympatho-vagal coactivation induced by torsadogenic molecules is elicited by reflex compensatory mechanisms in response to changes in stroke volume or cardiac output related to hemodynamic off-targets and/or QT prolongation. QTc prolongation was concealed or markedly blunted by the sympathetic component activation in a large proportion of tested torsadogenic drugs. Sympathetic reflex mechanisms in LQT patients similar to that found for dofetilide was also revealed in both patients exhibiting QTc prolongation and concealed QTc prolongation, irrespective to LQT type. Conclusions and implications: QTc prolongation and/or drug-induced hemodynamic side effects enhance beat to beat ventricular repolarisation variability via sympatho-vagal reflex compensatory mechanisms. Considering the sympathetic reflex component via analysis of HFQT oscillations dramatically improves prediction, sensitivity and specificity of drug induced Torsades de pointes risk assessment.
\end{abstract}

\section{Introduction}

Torsades de Pointes (TdP) are a malignant form of ventricular arrhythmia that can degenerate into ventricular fibrillation and sudden cardiac death. This particular kind of arrhythmia is commonly related to a lengthening of ventricular repolarisation. Consequently, the increase in ventricular repolarisation duration by drug-candidates is a major issue since it is associated with the risk of TdP (Kannankeril et al., 2010). Such QT prolongation has been strongly linked to cardiac hERG channels blocking properties since hERG channels are responsible for an inwardly rectifying outward potassium current, $\mathrm{I}_{\mathrm{KR}}$, that largely contributes to action potential repolarisation of ventricular cardiomyocytes. Despite the identification of this mechanism, prediction of the risk for QT prolongation remains uncertain during the preclinical development from hERG blocking properties and QT studies (Park et al., 2018). Similar difficulties are met in the clinic to identify at risk patients affected by Long QT Syndromes (LQTs). Indeed, $25 \%$ approximately of genotyped LQTs patients do not exhibit signs of QT prolongation (Goldenberg et al., 2011). The beat to beat variability of ventricular repolarisation (BVR) also plays a major role in TdPs (Thomsen et al., 2004; Lengyel et al., 
2007). In sinusal rhythm, the greatest contributor to BVR is the parasympathetic nervous system through rate dependent mechanisms. Indeed, rhythmic vagal discharges in the High Frequency (HF) band $(>0.1 \mathrm{~Hz})$ are responsible for large beat to beat heart rate changes causing in turn an important beat to beat rate dependent adaptation of ventricular repolarisation duration. Suppression of HF oscillations of heart rate (HFHR) and QT interval (HFQT) by a ganglioplegic agent was found to fully prevent dofetilide induced TdPs in cynomolgus monkeys (Champéroux et al., 2015). In beagle dogs, magnitude of HFHR oscillations was found to be increased by several hERG blockers causing TdPs in human. Increases in HFQT oscillations induced by these hERG blockers through parasympathetic raise result from a concomitant sympathetic activation (Champéroux et al., 2016). Altogether, these studies have permitted identification of a particular state of the autonomic control characterised by a coactivation of both the parasympathetic and sympathetic systems named S2 oscillations (Champéroux et al., 2018). However, the mechanism by which torsadogenic hERG blockers causes this coactivation is still unclear. Sympathetic activation often results from reflex compensatory mechanisms. So, we focused on the hemodynamic effects of hERG blocking drugs and their consequences on HF oscillations to determine a possible utility of a refined integrative approach combining hemodynamic, electrophysiological and autonomic biomarkers to predict TdP risk. For this aim, we used a representative set of $20 \mathrm{hERG}$ blockers including 15 compounds incriminated in TdP. To our knowledge, it is the first time that a refined analysis of hemodynamic, electrophysiological and autonomic effects of 20 hERG blocking drugs was presented in one integrative study. Thus, these observations may also serve to future researches on the topic of evaluation and management of TdP risk .

\section{Methods}

\section{Telemetric recordings}

Twenty-four-hour telemetric ECG and arterial pressure recordings were collected in beagle dogs. All animal experiments were subjected to ethical review (ethics committee $n^{\circ}$ CEEA-111) according to European directive 2010/63/UE on animal welfare. Reporting of experiments follows the ARRIVE guidelines (Kilkenny et al ., 2010; McGrath and Lilley, 2015). This work complies with all of the requirements of the BJP's Declaration on Experimental Design and Analysis. In accordance with the 3Rs encouraging the reduction in the number of animals used for experimental research, experiments already recorded in the internal ERBC database from 2008 were re-analyzed for this work. These reanalyses addressed the following compounds: cisapride, dofetilide (high dose), haloperidol, moxifloxacin (high dose), quinidine, risperidone, sotalol, terfenadine and thioridazine. Experiments specifically conducted for this study addressed the following compounds: astemizole, chlorpromazine, dofetilide (low dose), droperidol, ibutilide, milrinone, moxifloxacin (high dose), pimozide, prazosin and sertindole. All animal experiments were conducted in the following conditions described thereafter. Adult ( 3 males and 3 females per group for former studies or 6 males per group for recent studies due to logistic reasons linked to group housing) beagle dogs (10-15 kg, 8-24 months, CEDS, Mezilles, France) were fitted with radio telemetry transmitters (TL11M2D70PCT, L11 or M11 models, Data Sciences International, Saint Paul, USA). Dogs were premedicated with acetylpromazine (0.05 mg/kg, s.c.) and buprenorphine $(0.01 \mathrm{mg} / \mathrm{kg}$, s.c. $)$. Anaesthesia was induced by thiopental $(15-20 \mathrm{mg} / \mathrm{kg}$, i.v.) and then maintained with isoflurane $0.5-1.5 \%$ in oxygen. After left thoracotomy, one electrode was sutured directly to the left ventricular epicardium near the apex while the second electrode was sutured to the pericardium above the right atrium to approximate a limb Lead II ECG. Arterial pressure sensor was introduced via the femoral artery up to the caudal portion of the abdominal aorta. Analgesic treatment with buprenorphine/meloxicam was continued for a minimum of 2 days to alleviate any post-operative pain. A minimum period of 3 weeks was allowed for recovery from the surgery. For experiments conducted for the present study, animals were housed in pens by groups of two to four animals at maximum with playing tools. Environmental parameters were recorded continuously and maintained within a fixed range, room temperature at $15-21^{\circ} \mathrm{C}$ at $45-65 \%$ relative humidity. The artificial day/night cycle was $12 \mathrm{~h}$ light and $12 \mathrm{~h}$ darkness with light on at 07:30 am. Drinking water was provided ad libitum. Solid diet (300 g/animal) was given daily in the morning. All dosing with drugs was performed between 2:00 and 3:30 pm. within less than 30 minutes in each study. Arterial pressure and ECGs were recorded continuously for a minimum of $2 \mathrm{~h}$ before dosing up to $24 \mathrm{~h}$ post dose. Animals serve as their own control according to a cross-over design with a washout period of 48 to 72 
hours between dosing sessions. ECG and blood pressure (BP) signals were recorded at a sampling rate of $500 \mathrm{~Hz}$ using $\mathrm{ART}^{\mathrm{TM}}$ acquisition software (release 4.33, Data Sciences International, St Paul, MN, USA) or Ponemah $^{\mathrm{TM}}$ (release 6.33, Data Sciences International, St Paul, MN, USA).

\section{Human ECGs}

Twenty-four-hour Holter recordings (2/3-lead configuration, sampling rate: $200 \mathrm{~Hz}$ ) were obtained from the Telemetric and Holter ECG Warehouse (THEW, hosted at the University of Rochester Medical Center, NY, USA), an initiative hosting a warehouse of digital Holter ECGs open to the scientific community (Couderc 2012). The E-HOL-03-0202-003 database of 200 healthy subjects (age >15: 39 $\pm 16,100$ females and 100 males) was analysed. Healthy subjects were considered as eligible for enrollment and defined as healthy based on a battery of tests and information including no history of cardiovascular disease or disorders (stroke, TIA, peripheral vascular disease), no hasty of high $\mathrm{BP}(>150 / 90 \mathrm{mmHg})$, no medication, no chronic illness, normal physical examination, normal echocardiography, normal exercise testing and no pregnancy. The E-HOL-030480-013 database of genotyped LQTS patients was analysed for comparison with healthy subjects. Holter recordings were analysed from 104 untreated LQT1 patients (age > 15: 38 $\pm 15,60$ females and 54 males) and 35 untreated LQT2 patients (age $>15: 37 \pm 15,16$ females and 19 males).

\section{Beat to beat analysis}

Beat-to-beat heart rate, diastolic (DAP), mean and systolic (SAP) arterial pressures were calculated from the BP signal using software developed in RPL (RS/1 programming language, RS/1 release 6.3, Applied Materials). RR interval (ms) was derived from beat to beat $\mathrm{HR}$ values: $\mathrm{RR}=60 / \mathrm{HR}^{*} 1000$.

\section{HF oscillations}

The term "HF oscillations" refers to the largest magnitude of beat-to-beat heart rate (HFHR), heart period (HFRR) and QT interval (HFQT) changes measured at maximum and minimum of oscillations within discrete 10-second intervals.

\section{HFAM model}

The HFAM model was demonstrated to provide reliable assessment of the autonomic balance of the heart rate control in beagle dogs, cynomolgus monkeys and humans over short 10-second recording sequences (Champéroux et al., 2018). This model is based on the principle that HF oscillations are not stationary and is applicable to ambulatory and freely moving conditions. In the opposite, spectral analysis in the frequency domain requires stationary rhythms collected at rest in controlled breathing conditions (Task force, 1996).

HFHR and HFRR oscillations were normalized (N) as follows:

$\mathrm{HFHRN}=\mathrm{HFHR} / \mathrm{HFHR}_{\text {ref }}$ in normalized units

$\mathrm{HFRRN}=\mathrm{HFRR} / \mathrm{HFRR}_{\text {ref }}$ in normalized units

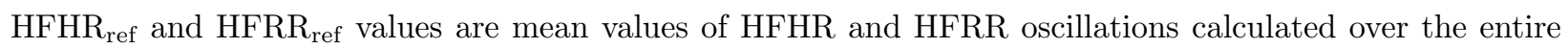

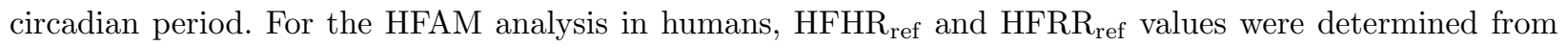
population values $\left(\mathrm{HFHR}_{\text {ref }}=10 \mathrm{bpm}\right.$ and $\left.\mathrm{HFRR}_{\mathrm{ref}}=110 \mathrm{~ms}\right)$. In dogs, these reference values were calculated individually for each animal during a free-treatment 24-hour recording session preceding each experiment.

The HFAM ratio was calculated from the following ratio: HFAM=HFHRN/HFRRN.

The HFAM model allows differentiation of three states of the autonomic control named S1, S2 and S3. Discrete algorithms were designed to automatically identify each state within each 10 -second recording sequence:

S1: HFAM[?]1.

S2: HFAM $>1$ and HFHRN $>1$.

S3: $\mathrm{HFAM}>1$ and $\mathrm{HFHRN}<1$. 
These states were demonstrated to identify 10-second sequences with parasympathetic predominance (S1), parasympathetic and sympathetic coactivation (S2) and parasympathetic withdrawal and/or sympathetic activation (S3). Proportions of each state were calculated in $\%$ per hour.

\section{Pulse contour model}

Stroke volume (SV) was determined from the following algorithm: SV=k.AUCsys where AUCsys is the area under curve of the arterial pressure signal from the DAP (start of systolic ejection) up to the start of the dichrotic wave (closure of sigmoid valves). Its principle is based on the proportionality of AUCsys to the SV (Kouchoukos et al., 1970). This model has been demonstrated to provide accurate and reliable results for SV assessments versus the thermodilution technique in anaesthetised dogs (Briganti et al., 2018). Since the location at which arterial pressure is measured might influence the reliability of the model, a thorough validation was conducted in anesthetised $\operatorname{dogs}(\mathrm{n}=6)$ by placing the tip of the pressure sensor of telemetric transmitter at the same location as in experiments conducted in conscious dogs, i.e. the caudal portion of the abdominal aorta. Reliability and pharmacological robustness of the model were tested in anaesthetised dogs following various pharmacological challenges expected to induce changes in SV: nitroprusside ( $30 \mu \mathrm{g} / \mathrm{kg}$, iv), phenylephrine $(10 \mu \mathrm{g} / \mathrm{kg}$, iv), dobutamine $(30 \mu \mathrm{g} / \mathrm{kg}$, iv), verapamil $(100 \mu \mathrm{g} / \mathrm{kg}$, iv) and hexamethonium $(1$ $\mathrm{mg} / \mathrm{kg}$, iv). The Pulse Contour model was compared to the ultrasonic Doppler flow method as reference method. Ultrasonic probes (PAX transit time ultrasonic flow probes and Ultrasonic transit-time flowmeter T402, Transonic Systems, NY, USA ) were placed around the ascending aorta close to the sigmoid valves. The pulse contour model has never been published in the context of telemetry in beagle dogs. So, results of the validation study are presented as supplemental material 1 . This validation demonstrated the reliability and pharmacological robustness of the pulse contour model in healthy dogs when compared to the ultrasonic flow method whatever the pharmacological challenges (bias: $+0.1 \mathrm{~mL}$, agreement: $\pm 1.5 \mathrm{~mL}$ according to Bland \& Altman analysis).

\section{Statistical procedures}

Comparisons between groups were performed using an analysis of variance for repeated measures and Dunnett's test. The level of probability $(\mathrm{P})$ deemed to constitute the threshold for statistical significance was fixed to $\mathrm{P}[?] 0.05$. Results are expressed as mean values +- SEM. The exact group size (n) is provided in legends of tables and figures. "n" values refer to independent animals or subjects and not replicates. Statistics were performed using RS/1 release 6.3, Applied Materials. Operators for animal experiments and data analysts were not blinded. This work does not contain any subjective observation or analyses that would justify blinding. Furthermore, all calculations were made by using fully automated computer procedures (GLP validated) from beat to beat analysis up to statistics.

\section{Drugs and choice of doses}

Molecules were purchased from Abcam Biochemicals (Abcam Biochemicals, Cambridge, United Kingdom), Biotrend (BioTREND, Chemikalien GmbH, Koln, Germany), Carbosynth (Carbosynth Ltd, Berkshire, United Kingdom), Clinisciences (CliniSciences, Nanterre, France), Sequoia Research Product ltd (Sequoia Research Products, Pangbourne, United Kingdom) and Sigma-Aldrich (Saint Quentin, France). The list of pharmacological agents used for validation of the pulse contour model is provided in Supplemental Material 1, Table 1. The full list of tested drugs including suppliers, vehicles and volumes of administration depending on dose levels and route administration is provided in Supplemental Material 2, Table 2. Doses were selected from preliminary trials to determine the minimum effective dose causing either QTc prolongation, increase in HFQT oscillations or change in autonomic control. A set of experiments were also conducted at higher doses with dofetilide, moxifloxacin, quinidine, sotalol and thioridazine.

\section{Nomenclature of targets and ligands}

Key protein targets and ligands in this article are hyperlinked to corresponding entries in http://www.guidetopharmacology.org, the common portal for data from the IUPHAR/BPS Guide to PHARMACOLOGY (Harding et al., 2018), and are permanently archived in the Concise Guide to PHARMACOL- 
OGY 2017/18 (Alexander et al., 2017).

\section{Results}

Kinetic of drug induced changes over the 24-hour post dosing recording period in all analysed parameters is shared as supplemental material 2. It includes a set of 20 hERG blocking drugs. Effects of 15 hERG blocking drugs with clinical reports of TdP (Redfern et al., 2003) are reported at the first dose level causing either QTc prolongation and/or increase in HFQT oscillations. This database also reports the effects of 5 non torsadogenic hERG blockers (including prasozin), milrinone and 6 other drugs interacting with the autonomic nervous system (atenolol, atropine, clonidine, hexamethonium, isoproterenol, phenylpherine, Supplemental Figures 106 to 126).

\section{Overview of drug induced hemodynamic, autonomic and electrophysiological effects}

Table 1 allows differentiating two main profiles among torsadogenic drugs at the first dose level causing either QTc prolongation and/or increase in HFQT oscillations. The first profile corresponds to drugs causing non blunted QTc prolongation. All these drugs showed a rapid onset in QTc prolongation within 1-2 hours after dosing. All the other torsadogenic drugs exhibited a profile characterised by blunted QTc prolongation or no QTc prolongation at the first dose showing an increase in HFQT oscillations. Blunted QTc prolongation was characterised by a delayed onset in QTc prolongation and delayed peak of QTc prolongation. 13 out of 15 torsadogenic hERG blocking drugs induced increases in HFQT oscillations. In addition, 13 experiments were conducted in combination with atenolol, a $\beta$-adrenoceptor blocking drug. Two non-torsadogenic drugs were also found inducing increases in HFQT oscillations and reported in the list for comparison: prasozin, an $\alpha 1$ adrenoceptors competitive antagonist and milrinone, a phosphodiesterase 3 inhibitor. Four non torsadogenic hERG blockers (ciprofloxacin, ranolazine, ebastin, verapamil) presented no QTc prolongation and no increase in HFQT oscillations at the first dose levels showing cardiovascular effects and/or changes in autonomic control.

\section{Torsadogenic hERG blockers combined with non blunted QTc prolongation}

6 torsadogenic hERG blockers caused QTc prolongation without evidence of blunting of QTc prolongation. 2 of them (moxifloxacin and quinidine) were found to induce a mild QTc prolongation only at a low dose. No autonomic and hemodynamic changes were seen with these two drugs at this low dose. Alternatively, 4 hERG blockers (astemizole, dofetilide, ibutilide, sotalol) caused larger QTc prolongation combined with signs of autonomic control changes and increases in HFQT oscillations. No apparent changes in hemodynamic parameters were observed in parallel. In this group, autonomic variations involved increases in S1 and/or S2 oscillations and reciprocal decrease in S3 oscillations depending on drugs and dose level. Increases in S1 oscillations seen with sotalol are consistent with its $\beta$ adrenoceptors blocking properties. For the 3 other hERG blockers causing QTc prolongation, changes in autonomic control could reflect compensatory mechanisms aimed to counterbalance direct hemodynamic effects. The only detected hemodynamic change being possibly responsible for triggering a baroreflex response was an increase in SV induced by dofetilide at a high dose under $\beta$ adrenoceptors blockade (Figure 1). As a reminder, atenolol is fully devoid of hemodynamic effect in healthy dogs causing a lowering in HFHR and S2 oscillations only (Supplemental Figures 106 to 108) due to the suppression of the coactivation mode of the autonomic control (Champéroux et al., 2016). This effect of dofetilide on SV under $\beta$ adrenoceptors blockade was independent on mean heart rate, this latter parameter being unchanged between control and the dofetilide+atenolol combination sessions during the period where SV was increased by dofetilide. Such an effect of dofetilide on SV could be the trigger for a baroreflex parasympathetic activation since this kind of effect is expected inducing an increase in cardiac output and BP in the absence of this compensatory baroreflex response. To better comprehend the relationship between the effect seen with dofetilide at a high dose on SV and the activation of the sympathetic component during S2 oscillations, changes in BP and SV were investigated during S2 oscillations. As shown from the Figure 2A obtained in a control dog, large drops in DAP occur during the deceleration phase within 1 to 2 beats only during S2 oscillations. They are associated with an increase in SV showing a greater left ventricle filling during deceleration. Drops in DAP reach up to $-50 \mathrm{mmHg}$ or more during $\mathrm{S} 2$ oscillations and 
are followed by compensatory increases in DAP of the same magnitude during acceleration phases to maintain constant mean DAP levels during HF oscillations. Building the DAP/HFHR and SV/HFHR relationships at peak and minimum heart rate during HF oscillations (Figure 2) shows that this reflex mechanism contributes to maintain almost constant mean DAP and SV levels during HF cycles. Interestingly, the largest HF oscillations of systolic arterial pressure (HFSAP) are much less pronounced than HFDAP oscillations. Taken together, these findings strongly support that this state of sympathetic and parasympathetic coactivation results from a reflex sympathetic activation during parasympathetic driven oscillations aimed to maintain constant mean DAP levels during HF cycles when parasympathetic activity is increased in response to an increase in SV and/or a decrease in DAP at the end of the deceleration phase. Besides, dofetilide induced effect on SV appears related to the magnitude of QT prolongation. Indeed, this effect was detected only at a high dose of dofetilide (Supplemental Figures 91 to 93) causing a large QT prolongation, likely because of its additional property of late INa enhancement (Yang $\mathrm{T}$ et al., 2014). At a lower dose, dofetilide induced QTc prolongation was less important and $\beta$ adrenoceptors blockade did not reveal any increase in SV (Supplemental Figures 13 to 15). Likewise, low level of QTc prolongation such those induced by moxifloxacin (Supplemental Figures 1 to 3) and quinidine (Supplemental Figures 4 to 6) did not trigger this compensatory reflex suggesting that the magnitude of QTc prolongation caused by these drugs at a low dose was not large enough to cause this hemodynamic effect on SV and trigger this reflex mechanism. At a higher dose, these two drugs induced larger QTc prolongation (Supplemental material, Figure 94 to 99). However, they also caused large decrease in SV combined with lowering in cardiac output. Blunted S3 oscillations were largely increased suggesting a more important sympathetic activation in response to these hemodynamic effects at high dose. This hemodynamic profile of moxifloxacin and quinidine at a high dose characterised by a lowering in cardiac function efficiency (cardiac output and SV) is consistent with their sodium and calcium channel blocking properties (Le Guennec et al., 2016).

\section{Torsadogenic drugs combined with blunted QTc prolongation or concealed QTc prolongation}

In the opposite to drugs causing QTc prolongation, all the other torsadogenic hERG blocking showed signs of blunted QTc prolongation or no QTc prolongation. In parallel, they all showed signs of uncompensated hemodynamic effects associated with an autonomic coactivation and increases in HFQT oscillations. The term "uncompensated hemodynamic effects" means that changes in hemodynamic parameters are visible and was not maintain unchanged by the autonomic reflex mechanisms. For six of them (chlorpromazine, droperidol, haloperidol, risperidone, sertindole and thioridazine), this pattern was associated with a sustained decrease in SV (Figure 3). Since all these latter drugs share alpha-1 adrenoceptors blocking properties (Nedergaard et al., 1988; Sleight et al., 1993; Nourian et al., 2008), their profile was compared to a selective alpha-1 adrenoceptors blocker, prazosin. This drug was found causing the same profile of lowering in SV with the same consequences in terms of autonomic coactivation and increases in HFQT oscillations (Figure 3). Milrinone, a phosphodiesterase 3 inhibitor, also caused an autonomic coactivation related to lowering in SV associated with an increase in HFQT oscillations (Figure 3) like prazosin and torsadogenic alpha-1 adrenoceptors and hERG blocking drugs. Decrease in SV is attributed to peripheral vasodilation resulting from phosphodiesterase inhibition in vascular smooth muscles. Interestingly, milrinone caused QTc shortening (Supplemental Figures 70 to 72) showing that this increase in ventricular repolarisation variability (HFQT) can occur even when ventricular repolarisation is shortened. According to the reflex mechanism described earlier inside HF oscillations, this lowering in SV related to alpha-1 adrenoceptors blockade or phosphodiesterase inhibition could be the trigger for a sympathetic activation followed by compensatory parasympathetic activation to maintain stable mean SV and DAP levels during HF oscillations. Conversely, cisapride and pimozide caused both a mild increase in BP combined with an increase in cardiac output (Figure 4). As stated earlier for dofetilide, this kind of hemodynamic effect is expected to induce a baroreflex parasympathetic activation with the similar reflex consequences during the HF cycles. These data further support the concept according which these oscillatory mechanisms are aimed to maintain stable mean DAP during the HF cycles in case of mild drug induced lowering or increase in SV. Unlike torsadogenic alpha-1 adrenoceptors blocking drugs, off-target(s) responsible for this particular profile remain unidentified from the literature for pimozide and cisapride. 
In the opposite to hERG blocking drugs causing non blunted QTc prolongation, expected QTc prolongation was dramatically blunted or absent although all these molecules induced an increase in HFQT oscillations. QTc prolongation was revealed under conditions of $\beta$ adrenoceptors blockade (Figure 5) applied to 7 drugs of this group except for chlorpromazine. In other words, QTc prolongation was concealed for these 6 torsadogenic drugs. This also means that hemodynamic effects responsible for this sympathetic reflex mechanism appear at doses at which the ventricular repolarisation prolongation due to hERG blockade is low or absent (chlorpromazine) and can be concealed by a sympathetic activation. Reciprocally, drugs causing non blunted QTc prolongation are molecules devoid of hemodynamic effects due to off-targets at dose levels causing ventricular repolarisation prolongation. These findings demonstrate that this phenomenon of concealed QTc prolongation described earlier with thioridazine at a high dose (Champéroux et al., 2010) is shared by several other torsadogenic hERG channel blocking drugs (droperidol, risperidone, sertindole, pimozide and terfenadine). In the case of terfenadine at least, QTc prolongation is likely also blunted because of opposite electrophysiological properties on ventricular repolarization related to its hERG and calcium/sodium channels blocking profile (Champéroux et al., 2005). This is the second main mechanism responsible for concealed QTc prolongation. Terfenadine cumulates both phenomena.

\section{Lessons from LQT1 and LQT2 syndromes}

In addition of iatrogenic TdP, loss of function mutations on potassium repolarizing channels could lead to TdP in a mechanism supposed to involve QT prolongation in LQT1 and LQT2 syndromes. Nevertheless, despite of confirmed mutations, QTc prolongation is concealed in $25 \%$ of these patients approximately (Goldenberg et al., 2011). In the present study, patients with concealed QTc prolongation was characterised by a QTc $<450$ $\mathrm{ms}$ in males and QTc $<470 \mathrm{~ms}$ in females using the Bazett formula (Page et al., 2015). To tempt to decipher impact of mutations on HFAM biomarkers of TdP risk depending on magnitude of QTc prolongation, we analysed ECGs from the THEW cohort of untreated LQT1 and LQT2 patients with QTc prolongation and concealed QTc prolongation compared to healthy individuals (Table 2). Applying the HFAM model (Supplemental Figures 127 to 134) shows higher sympathetic activity level (increase in S3) and HFQT oscillations in patients with QTc prolongation than in patients with concealed QTc prolongation in both types of LQT syndrome (Figure 6). This phenomenon occurs during the light period of the circadian cycle where patients are assumed to be majorly in standing up position. The S2 oscillations proportion in patients with QTc prolongation tended also to be higher but the difference with concealed QTc prolongation patients did not reach the statistical significance threshold. This global increase (S2+S3) in sympathetic activity appears with both mechanisms responsible for QT prolongation, i.e. decrease in $\mathrm{I}_{\mathrm{Ks}}$ current for LQT1 and decrease in $\mathrm{I}_{\mathrm{Kr}}$ current in LQT2 respectively. Since magnitude of HFHR oscillations were unchanged (Table 2) in both LQT syndromes when compared to patients with concealed QTc prolongation, the increase in HFQT oscillations seen in patients with QTc prolongation is rather attributed to increase in sympathetic activity according to the vertical shift mechanism of the HFHR/HFQT relationship as described earlier for torsadogenic hERG blockers (Champéroux et al., 2016). Comparison of subgroups of patients exhibiting concealed QTc prolongation to the healthy subjects' sample also provides interesting information. Indeed, despite no noticeable difference in QTc, QT interval is prolonged both in LQT1 and LQT2 patients when compared to healthy subjects. This difference is related to lower heart rate levels (Table 2) due to an increase in parasympathetic activity (increase S1 oscillations and lowering in HFAM ratio). In parallel, HFQT oscillations are increased over the whole circadian cycle. Like dofetilide at low and high dose, an increase in parasympathetic activity is mainly observed for lower level of QT/QTc prolongation whereas sympathetic activity is increased depending on magnitude of QT prolongation in both LQT syndrome types.

\section{Discussion}

This set of 15 hERG blocking drugs reported as causing TdP includes 9 of the most potent hERG blockers of the Kramer list (Kramer et al., 2013). All these torsadogenic drugs induced hemodynamic effects activating in turn autonomic reflex mechanisms. These hemodynamic effects were different depending on drug and dose levels. In most of cases, they were more or less compensated by reflex mechanisms making them difficult to detect directly from blood pressure changes only. In parallel, combining QTc prolongation and 
HFQT oscillations analysis allowed detection of all tested torsadogenic drugs since visible from at least one of these two biomarkers. In the absence of QTc prolongation, enhancement of HFQT oscillations also constitutes a sensitive surrogate for suspicion of possible concealed QTc prolongation at low doses of torsadogenic hERG blockers. This paradigm is due to specific off-targets which conceal the hERG blockade induced QTc prolongation by activating the sympathetic system during HF cycles. This phenomenon likely involves the recruitment of the $I_{K s}$ repolarisation reserve by $\beta$-adrenoceptors mediated sympathetic activation (Volders et al., 2003). The $\alpha 1$ adrenoceptors blocking property is by far the most common off-target among torsadogenic drugs. This is not the only one since drugs like pimozide and cisapride also induced autonomic coactivation while they were not reported as exhibiting this $\alpha 1$ adrenoceptors blocking property. The milrinone profile shows that phosphodiesterase inhibition could also constitute an off-target leading to an autonomic coactivation and increase in HFQT oscillations. This example is important because it demonstrates that increase in HFQT oscillations do not require necessarily any mechanism lengthening the ventricular repolarisation since milrinone is devoid of hERG blocking properties and even causes QTc shortening. First of all, this work demonstrates that HFQT oscillations magnitude is a sensitive biomarker of the sympathetic activity on autonomic beat to beat variability of ventricular repolarisation. This biomarker is particularly useful when the parasympathetic system activity is predominant as with dofetilide at a low dose or in LQT patients with concealed QT prolongation. This dataset of hERG blockers also shows that this biomarker is more sensitive for detection of drug torsadogenic profile than short term QT variability that rather reflects instability of ventricular repolarisation in its principle.

Specificity for TdP risk assessment appears promising too. Indeed, increases in HFQT oscillations were found with torsadogenic hERG blockers only whereas non torsadogenic hERG blockers did not increase HFQT oscillations. However, two non arrhythmic drugs, prazosin and milrinone, were found sharing this ability to enhance this biomarker. Investigating available data from literature about sudden cardiac death risk reveals that milrinone (Packer et al, 1991), prazosin and other $\alpha 1$ adrenoceptors antagonists (O' Connell et al., 2014) were reported to unexplainably worsen mortality in patients with heart failure. Despite its hERG blocking properties (Thomas et al., 2004), we failed to find clinical reports of Tdp induced by prazosin. Conversely, milrinone was recently cited as involved in TdP events in humans (Chiba et al., 2020) although devoid of hERG blocking properties (Yunomae et al., 2007) and considered as a non torsadogenic drug. Milrinone was also reported as causing cardiotoxicity resulting from adrenergic hyperactivity (Chiba et al., 2020). Their propensity to cause excess mortality in the context of heart failure is certainly not a coincidence. Indeed, ventricular repolarization is lengthened in heart failure due to down regulation of potassium current (Näbauer \& Kääb, 1998) and enhancement of the late sodium current (Maltsev et al., 2007) creating as well favourable conditions for the triggering of ventricular arrhythmias by sympathetic activation as in LQT syndromes. These two examples of molecules open new perspectives for sudden cardiac death risk assessment out the area of TdP induced by arrhythmic drugs.

One of the most important finding in this work is the central role played by the stroke volume and cardiac output changes in reflex mechanisms leading to autonomic coactivation and increase in beat to beat ventricular repolarisation variability. This autonomic coactivation corresponds to an enhancement of oscillatory reflex mechanisms aimed to maintain stable the mean diastolic arterial pressure during HF parasympathetic cycles elicited by mild increases or decreases in stroke volume. Transient drops in diastolic arterial pressure following large RR pauses during HF cycles support involvement of the baroreflex in this autonomic coactivation phenomenon. However, mechanoreceptors sensitive to ventricular distension and volume could also be involved. They were shown to trigger large sympathetic burst following transient drop in arterial pressure in particular following premature ventricular contraction where the systolic pressure peak required to trigger arterial baroreceptors to terminate sympathetic outflow is absent (Zamir et al., 2012). Lowering in stroke volume induced by $\alpha 1$ adrenoceptors blocking drugs and other peripheral vasodilator drugs is considered as due to blood pooling in peripheral venous compartments below the diaphragm causing in turn a reduction in venous return and cardiac output (Brignole et al., 2018). Conversely, outcomes of dofetilide at a high dose under $\beta$-adrenoceptors blockade support that QT prolongation may result in a mild increase in stroke volume. Accordingly, positive inotropic effects correlated to their effects on ventricular repolarisation duration 
were reported for dofetilide and E4031 in ventricular muscle preparations (Tande et al., 1990; Abrahamsson et al., 1993).

This work establishes for the first time complex relationships between hemodynamic effects leading to sympathetic compensatory reflexes with arrhythmic electrophysiological mechanisms related to ventricular repolarisation prolongation. These relationships very well fit with the concept of the Coumel's triangle and allow to dramatically refining and update (Figure 8) the model based on the Coumel's triangle proposed earlier (Champéroux et al., 2015). According to this concept, TdP triggering involves $1 /$ a modulator: the autonomic nervous system: its contribution is featured by an enhancement of HF oscillations through a sympathetic coactivation in response to hemodynamic off-targets in case of vaso-active torsadogenic and/or due to ventricular repolarisation prolongation itself for drugs acting selectively on ventricular repolarization and in LQT syndromes, 2/ a substrate: the lengthening in ventricular repolarisation due to genetic mutations in LQT syndromes or related to intrinsic electrophysiological (off-)targets in case of torsadogenic drugs, 3/ a trigger: the sympathetic system that increases the probability of rate dependent arrhythmias under conditions of ventricular repolarization prolongation (Shimizu \& Antzlevitch, 1999) during acceleration phases of HF cycles or following large RR pause, reminding that $80 \%$ of TdP published in the clinical literature are pause dependent (Viskin et al., 2000).

Finally, two specific further features common to torsadogenic hERG blocking drugs, LQT1 and LQT2 syndromes should be emphasized. First, the magnitude of HFQT oscillations is increased in LQT1 and LQT2 syndromes as with most of torsadogenic hERG blockers causing QTc prolongation. In both cases, this enhancement of HFQT oscillations reflects an increase in sympathetic activity. In LQT syndromes, TdPs are mainly triggered in humans by sympathetic activation achieved in various situations such as physical exercice, swimming, fear reaction or arousal events (Schwartz et al., 2001, Kim et al., 2010, ). Like in LQT syndromes, these compensatory sympathetic reflexes caused torsadogenic drugs also appear as playing a key role for their arrhythmic profile. Besides, the proposed sympathetic reflex mechanisms provide a robust rational support to current therapeutic strategies applied in LQT syndromes based on use of $\beta$-blockers or sympathetic left stellate ganglia ablation (Priori et al., 2015). Secondly, drug induced QTc prolongation potency can be concealed as in LQT1 and LQT2 syndromes. This latter feature should have strong implications for preclinical safety pharmacology and drug safety. Indeed, the current preclinical strategy based on risk assessment of QT prolongation and hERG blockade showed a poor sensitivity in the 1x-10x exposure multiple range (Park et al., 2018). Combining QTc prolongation potency assessment, hemodynamic and autonomic modelling and HFQT oscillations analysis offers real opportunity for better estimation of drug safety margins and improving preclinical assessment of TdP risk and sudden cardiac death.

\section{References}

Abrahamsson C, Duker G, Lundberg C, Carlsson L. (1993). Electrophysiological and inotropic effects of H 234/09 (almokalant) in vitro: a comparison with two other novel IK blocking drugs, UK-68,798 (dofetilide) and E-4031. Cardiovasc Res 27:861-867.

Alexander SP, Striessnig J, Kelly E, Marrion NV, Peters JA, Faccenda E, ... Davies JA. (2017). The concise guide to pharmacology 2017/18: Voltage-gated ion channels. Br J Pharmacol, 174:S160-S194.

Brignole M, Moya A, de Lange FJ, Deharo JC, Elliott PM, Fanciulli A, .. van Dijk JG. (2018). ESC Guidelines for the diagnosis and management of syncope. Eur Heart J, 39:1883-1948.

Briganti A, Evangelista F, Centonze P, Rizzo A, Bentivegna F, Crovace A, Staffieri F. (2018). A preliminary study evaluating cardiac output measurement using Pressure Recording Analytical Method (PRAM) in anaesthetized dogs. BMC Vet Res, 14:72.

Champéroux P, Viaud K, El Amrani AI, Fowler JS, Martel E, Le Guennec JY, Richard S. (2005). Prediction of the risk of Torsade de Pointes using the model of isolated canine Purkinje fibres. Br J Pharmacol, 144:376385.

Champéroux P, Ouillé A, Martel E, Fowler JS, Maurin A, Jude S, .. Richard S. (2010). Interferences of 
the autonomic nervous system with drug induced QT prolongation: a point to consider in non-clinical safety studies. J Pharmacol Toxicol Methods, 61:251-263.

Champéroux P, Thireau J, Jude S, Laigot-Barbé C, Maurin A, Sola ML, .. Le Guennec JY. (2015). Dofetilide induced QT interval short term variability and ventricular arrhythmias are dependent on high frequency autonomic oscillations. Br J Pharmacol, 172:2878-2891.

Champéroux P, Le Guennec JY, Jude S, Laigot C, Maurin A, Sola ML, ... Thireau J. (2016). The high frequency relationship: implications for torsadogenic hERG blockers. Br J Pharmacol, 173:601-612.

Champéroux P, Fesler P, Judé S, Richard S, Le Guennec JY, Thireau J. (2018). High-frequency autonomic modulation: a new model for analysis of autonomic cardiac control. Br J Pharmacol, 175:3131-3143.

Chiba K, Ishizaka T, Yoshimatsu Y, Mikamoto K, Maeda Y, Iguchi T, ... Mori K. (2020). Comprehensive analysis of cardiac function, blood biomarkers and histopathology for milrinone-induced cardiotoxicity in cynomolgus monkeys. J Pharmacol Toxicol Methods. 103:106870. doi: 10.1016/j.vascn.2020.106870. Epub 2020

Couderc JP (2012). The Telemetric and Holter ECG Warehouse (THEW): the first three years of development and research. J Electrocardiol, 45:677-683.

Goldenberg I, Horr S, Moss AJ, Lopes CM, Barsheshet A, McNitt S, .. Zhang L. (2011). Risk for lifethreatening cardiac events in patients with genotype-confirmed long-QT syndrome and normal-range corrected QT intervals. J Am Coll Cardiol, 57:51-59.

Harding SD, Sharman JL, Faccenda E, Southan C, Pawson AJ, Ireland S, ... Davies JA. The IUPHAR/BPS Guide to PHARMACOLOGY in 2018: updates and expansion to encompass the new guide to IMMUNOPHARMACOLOGY. Nucl Acids Res 2018; 46: D1091-D1106.

Kramer J, Obejero-Paz CA, Myatt G, Kuryshev YA, Bruening-Wright A, Verducci JS, Brown AM. MICE models: superior to the HERG model in predicting Torsade de Pointes. Sci Rep, 3:2100.

Kannankeril P, Roden DM, Darbar D. (2010). Drug-induced long QT syndrome. Pharmacol Rev, 62:760-781.

Kilkenny C, Browne W, Cuthill IC, Emerson M, Altman DG (2010). NC3Rs Reporting Guidelines Working Group. Animal research: reporting in vivo experiments: the ARRIVE guidelines. Br J Pharmacol, 160: 15771579 .

Kim JA, Lopes CM, Moss AJ, McNitt S, Barsheshet A, Robinson JL, ... Goldenberg I. (2010). Triggerspecific risk factors and response to therapy in long QT syndrome type 2. Heart Rhythm, 7:1797-1805.

Kouchoukos NT, Sheppard LC, McDonald DA. (1970). Estimation of stroke volume in the dog by a pulse contour method. Circ Res, 26:611-23.

Le Guennec JY, Thireau J, Ouillé A, Roussel J, Roy J, Richard S, . . Champéroux P. (2016). Inter-individual variability and modeling of electrical activity: a possible new approach to explore cardiac safety? Sci Rep, $6: 37948$.

Lengyel C, Varró A, Tábori K, Papp JG, Baczkó I. (2007). Combined pharmacological block of I(Kr) and $\mathrm{I}(\mathrm{Ks})$ increases short-term QT interval variability and provokes torsades de pointes. Br J Pharmacol, 151:941951.

Maltsev VA, Silverman N, Sabbah HN, Undrovinas AI. (2007). Chronic heart failure slows late sodium current in human and canine ventricular myocytes: implications for repolarization variability. Eur J Heart Fail, 9:219-227.

McGrath JC, Lilley E (2015). Implementing guidelines on reporting research using animals (ARRIVE etc.): new requirements for publication in BJP. Br J Pharmacol, 172: 3189-3193. 
Moss AJ, Schwartz PJ, Crampton RS, Tzivoni D, Locati EH, MacCluer J, .. Choi S. (1991). The long QT syndrome. Prospective longitudinal study of 328 families. Circulation. 84:1136-1144.

Näbauer M, Kääb S. (1998). Potassium channel down-regulation in heart failure.

Cardiovasc Res, 37:324-334.

Nedergaard OA, Abrahamsen J. (1988). Effect of chlorpromazine on sympathetic neuroeffector transmission in the rabbit isolated pulmonary artery and aorta. Br J Pharmacol, 93:23-34.

Nourian Z, Mulvany MJ, Nielsen KB, Pickering DS, Kristensen T. (2008). The antagonistic effect of antipsychotic drugs on a HEK293 cell line stably expressing human alpha1A1-adrenoceptors. Eur J Pharmacol, 596:32-40.

O'Connell TD, Jensen BC, Baker AJ, Simpson PC. (2014). Cardiac alpha1-adrenergic receptors: novel aspects of expression, signaling mechanisms, physiologic function, and clinical importance. Pharmacol Rev, 66:30833.

Packer M, Carver JR, Rodeheffer RJ, Ivanhoe RJ, DiBianco R, Zeldis SM, Hendrix GH, ... DeMets DL. (1991). Effect of oral milrinone on mortality in severe chronic heart failure. The PROMISE Study Research Group. N Engl J Med, 325:1468-1475.

Page A, Aktas MK, Soyata T, Zareba W, Couderc JP. (2015). "QT clock" to improve detection of QT prolongation in long QT syndrome patients. Heart Rhythm, 13:190-198.

Park E, Gintant GA, Bi D, Kozeli D, Pettit SD, Pierson JB, ... Valentin JP. Can non-clinical repolarization assays predict the results of clinical thorough QT studies? Results from a research consortium. Br J Pharmacol, 175:606-617.

Priori SG, Blomström-Lundqvist C, Mazzanti A, Blom N, Borggrefe M, Camm J, ... Van Veldhuisen DJ. (2015). ESC Guidelines for the management of patients with ventricular arrhythmias and the prevention of sudden cardiac death: The Task Force for the Management of Patients with Ventricular Arrhythmias and the Prevention of Sudden Cardiac Death of the European Society of Cardiology (ESC). Eur Heart J, $36: 2793-2867$.

Redfern WS, Carlsson L, Davis AS, Lynch WG, MacKenzie I, Palethorpe S, ... Hammond TG. (2003). Relationships between preclinical cardiac electrophysiology, clinical QT interval prolongation and torsade de pointes for a broad range of drugs: evidence for a provisional safety margin in drug development. Cardiovasc Res 58:32-45.

Shimizu W, Antzelevitch C. (1999). Cellular basis for long QT, transmural dispersion of repolarization, and torsade de pointes in the long QT syndrome. J Electrocardiol, 32 Suppl:177-184.

Schwartz PJ, Priori SG, Spazzolini C, Moss AJ, Vincent GM, Napolitano C, .. Bloise R. (2001). Genotypephenotype correlation in the long-QT syndrome: gene-specific triggers for life-threatening arrhythmias. Circulation, 103:89-95.

Sleight AJ, Koek W, Bigg DC. (1993). Binding of antipsychotic drugs at alpha 1A- and alpha 1Badrenoceptors: risperidone is selective for the alpha 1B-adrenoceptors. Eur J Pharmacol, 238:407-410.

Tande PM, Bjørnstad H, Yang T, Refsum H. (1990). Rate-dependent class III antiarrhythmic action, negative chronotropy, and positive inotropy of a novel Ik blocking drug, UK-68,798: potent in guinea pig but no effect in rat myocardium. J Cardiovasc Pharmacol, 16:401-410.

Task Force of the European Society of Cardiology and the North American Society of Pacing and Electrophysiology. (1996). Heart rate variability: standards of measurement, physiological interpretation, and clinical use. Circulation, 93:1043-1065. 
Thomas D, Wimmer AB, Wu K, Hammerling BC, Ficker EK, Kuryshev YA, .. Karle CA. (2004). Inhibition of human ether-a-go-go-related gene potassium channels by alpha 1-adrenoceptor antagonists prazosin, doxazosin, and terazosin. Naunyn Schmiedebergs Arch Pharmacol, 369:462-472.

Thomsen MB, Verduyn SC, Stengl M, Beekman JD, de Pater G, van Opstal J, .. . Vos MA. (2004). Increased short-term variability of repolarization predicts d-sotalol-induced torsades de pointes in dogs. Circulation, 110:2453-2459.

Viskin S, Fish R, Zeltser D, Belhassen B, Heller K, Brosh D, ... Barron HV. (2000). Arrhythmias in the congenital long QTsyndrome: how often is torsade de pointes pause dependent? Heart, 83: 661-666.

Volders PGA, Stengl M, van Opstal JM, Gerlach U, Spätjens R, Beekman JDM, .. Vos MA. (2003). Probing the contribution of IKs to canine ventricular repolarization: key role for beta-adrenergic receptor stimulation. Circulation, 107:2753-2760.

Yang T, Chun YW, Stroud DM, Mosley JD, Knollmann BC, Hong C, Roden DM. (2014). Screening for acute IKr block is insufficient to detect torsades de pointes liability: role of late sodium current. Circulation,130:224234 .

Yunomae K, Ichisaki S, Matsuo J, Nagayama S, Fukuzaki K, Nagata R, Kito G. (2007). Effects of phosphodiesterase (PDE) inhibitors on human ether-a-go-go related gene (hERG) channel activity. J Appl Toxicol, 27:78-85.

Zamir M, Kimmerly DS, Shoemaker JK. (2012). Cardiac mechanoreceptor function implicated during premature ventricular contraction. Auton Neurosci , 167:50-55.

\section{Legends of tables}

Table 1. Summary table reporting hemodynamic, autonomic and electrophysiological effects of 20 hERG blockers (Supplemental Figures 1 to 105). All drugs were tested at the first dose level causing either QTc prolongation and/or increase in HFQT oscillations or change in autonomic control. 5 torsadogenic hERG blockers were tested at a higher dose (lower panel). Changes highlighted in black refer to changes that reached statistical significance threshold when compared to vehicle.

Table 2. Summary table reporting changes in autonomic biomarkers in LQT1 and LQT2 patients with QTc prolongation versus patients with concealed QTc prolongation and in LQT1 and LQT2 patients with concealed QTc prolongation versus healthy subjects. Supplemental Figures 127 to 134.

\section{Legends of figures}

Figure 1. Effects of astemizole $(1 \mathrm{mg} / \mathrm{kg}$, iv $)$, dofetilide $(0.1 \mathrm{mg} / \mathrm{kg}$, po) and dofetilide $(1 \mathrm{mg} / \mathrm{kg}$, po) alone or in combination with atenolol (1 mg/kg, iv) on QTc, stroke volume (SV), S1, S2 and/or HFQT oscillations in beagle dogs. Vehicle: red filled circles, drug alone: blue filled circles, drug combined with atenolol: blue empty circles. Data are presented as mean values $\pm S E M, n=6$.

Figure 2. (A) Example of S2 oscillations superimposed with BP signal showing large HF heart rate (HR) and stroke volume (SV) oscillations in a control dog. (B, C, D) Mean SV/HFHR, DAP/HFHR and SAP/HFHR relationships were built from mean values and from values recorded at maximum (HRhigh) and minimum (HRlow) of HR oscillations during all 10-seconds sequences recorded over the whole circadian cycle in control dogs. Data are presented as mean values \pm SEM, $n=6$.

Figure 3. Effects of thioridazine $(1.5 \mathrm{mg} / \mathrm{kg}$, po), prazosin $(1 \mathrm{mg} / \mathrm{kg}$, iv $)$ and milrinone $(3 \mathrm{mg} / \mathrm{kg}$, iv) on stroke volume (SV), S2 and HFQT oscillations in beagle dogs. Vehicle: red filled circles, drug alone: blue filled circles, drug combined with atenolol: blue empty circles. Data are presented as mean values \pm SEM, $\mathrm{n}=6$.

Figure 4. Effects of cisapride (6 mg/kg, po) and pimozide (1 mg/kg, iv) on and cardiac output (CO), S2 and HFQT oscillations in beagle dogs. Vehicle: red filled circles, drug alone: blue filled circles. Data are presented 
as mean values $\pm \mathrm{SEM}, \mathrm{n}=6$.

Figure 5. Examples of blunted QTc or concealed QTc prolongation revealed under $\beta$ adrenoceptors blockade by atenolol (1 mg/kg, iv) in dogs. Vehicle: red filled circles, drug alone: blue filled circles, drug combined with atenolol: blue empty circles. Data are presented as mean values $\pm \mathrm{SEM}, \mathrm{n}=6$.

Figure 6. Comparison of LQT1 and LQT2 patients with QTc prolongation (n=75 LQT1, n=26 LQT2) to patients with concealed QTc prolongation ( $n=29$ LQT1, $n=9$ LQT2) on QT interval, S3 and HFQT oscillations. Patients with concealed QTc prolongation: red filled circles, patients with QTc prolongation: blue filled circles. Data are presented as mean values \pm SEM.

Figure 7. Comparison of LQT1 and LQT2 patients with concealed QTc prolongation (n=29 LQT1, n=9 LQT2) to healthy subjects $(n=200)$ on QT interval, S1 and HFQT oscillations. Healthy subjects: red filled circles, patients with concealed QTc prolongation: blue filled circles. Data are presented as mean values \pm SEM.

Figure 8. Update of Coumel's triangle concept applied to torsadogenic drug and LQT (type 1 and 2) syndromes. Detailed explanations are provided in the last part of the discussion.

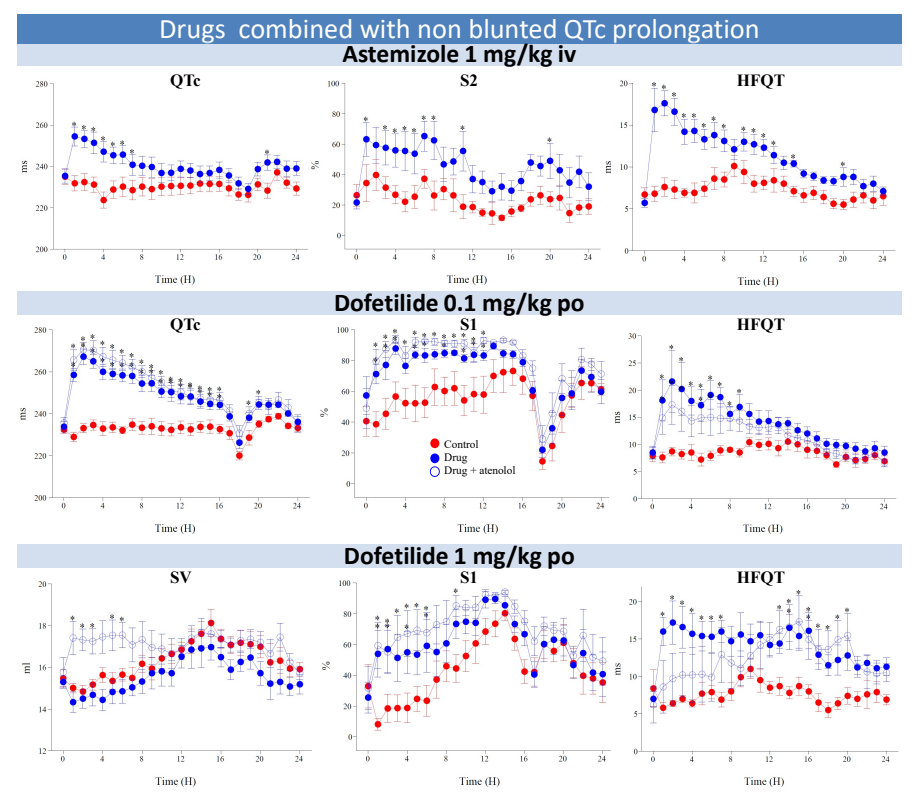


Beat to beat SV versus HR during HF oscillations

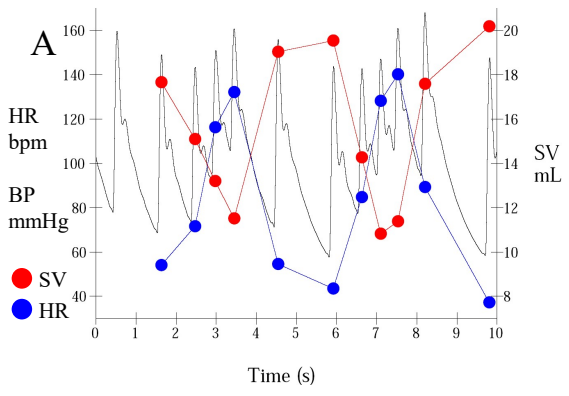

DAP/HFHR relationship

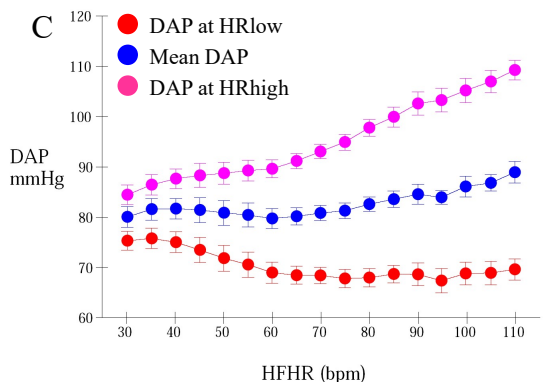

SV/HFHR relationship

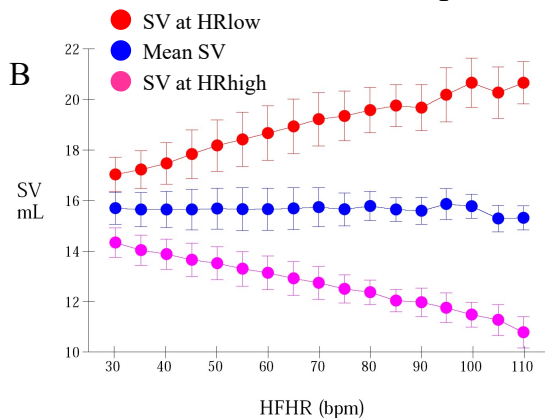

SAP/HFHR relationship

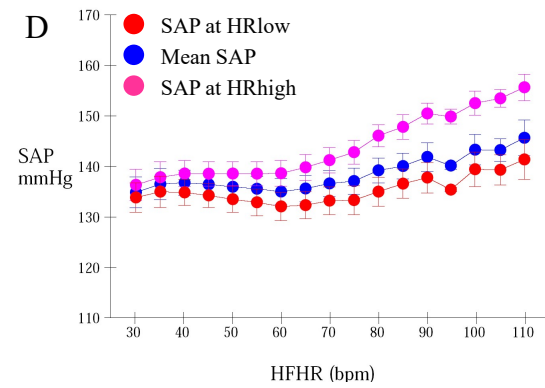

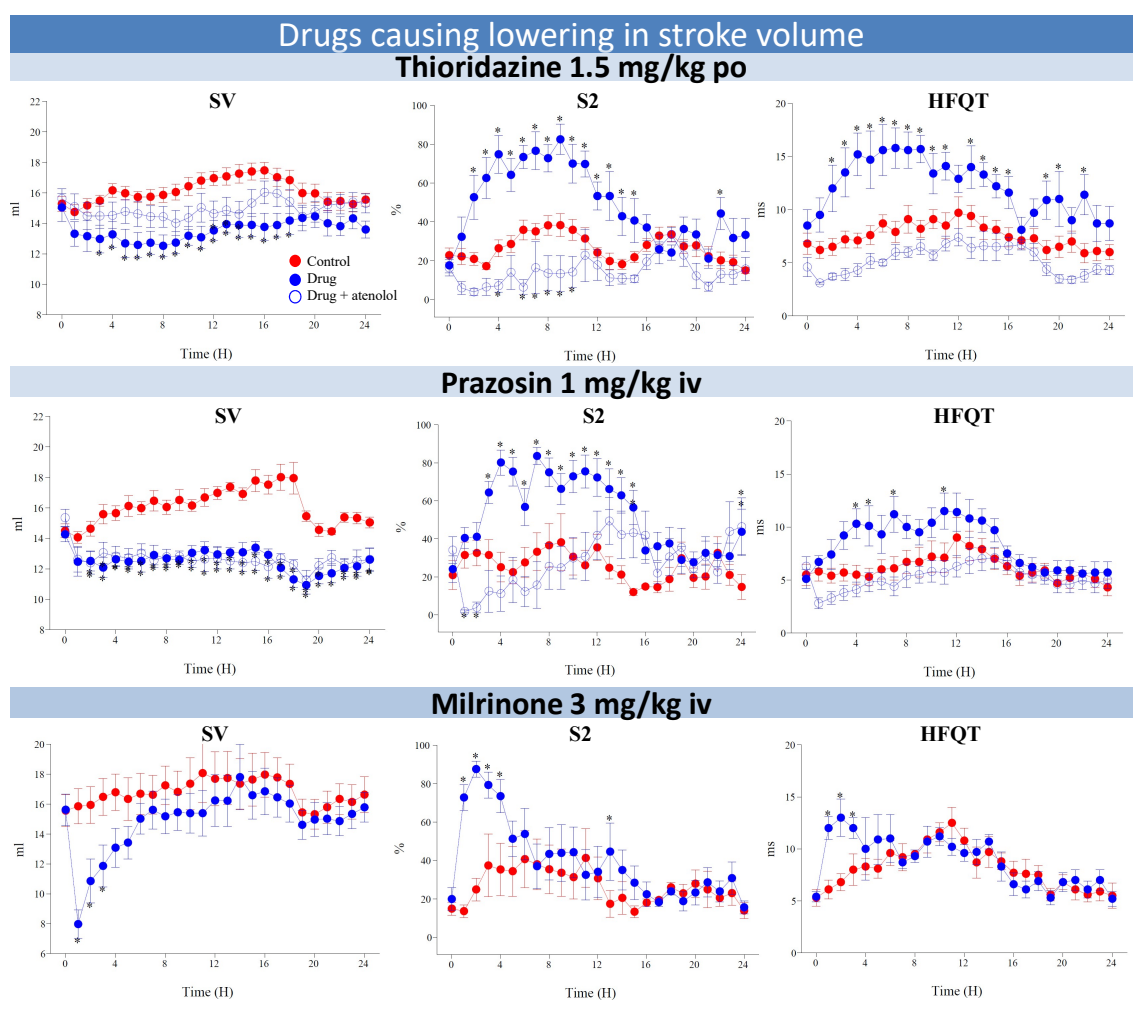



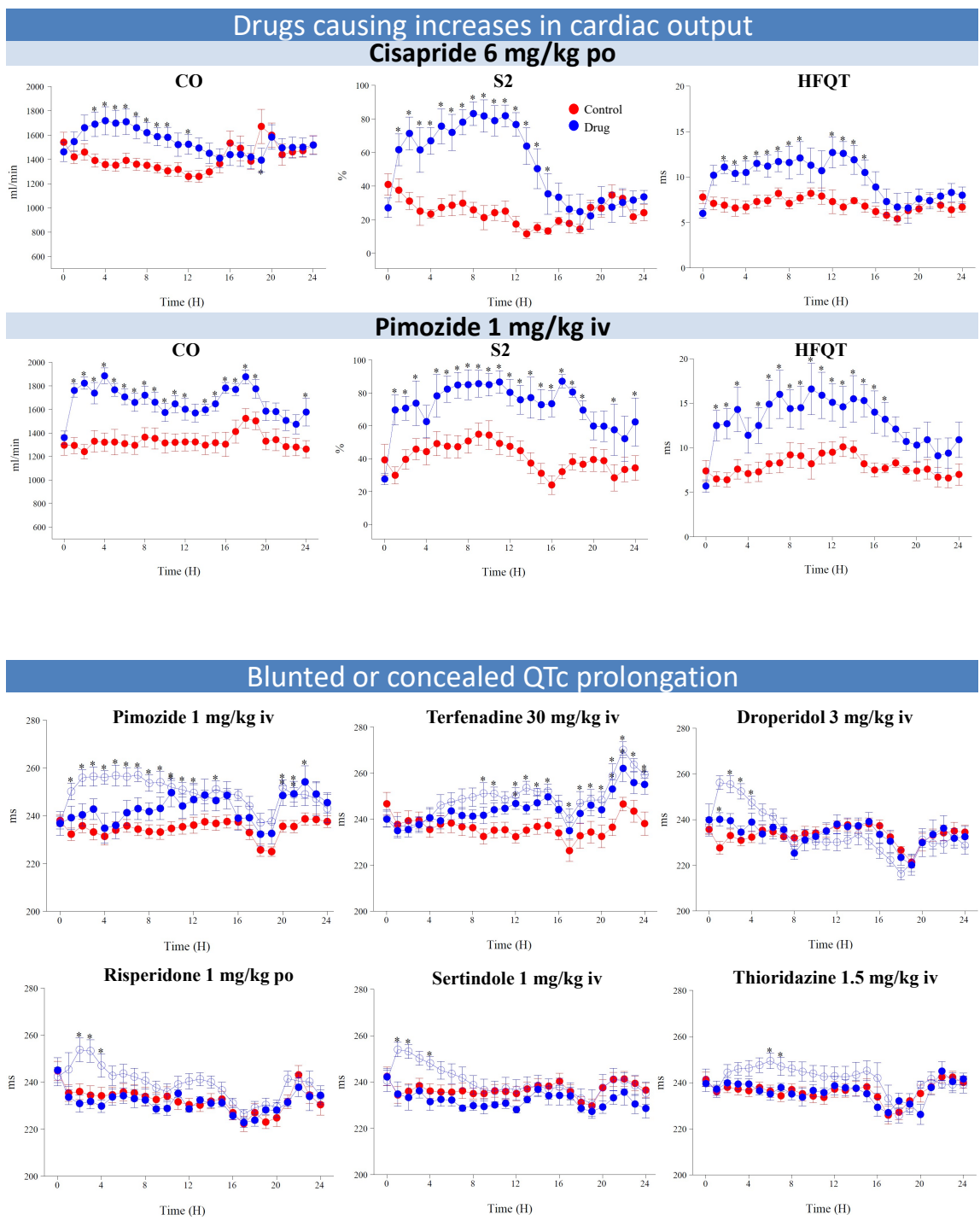
Patients with concealed QTc prolongation vs patients with QTC prolongation

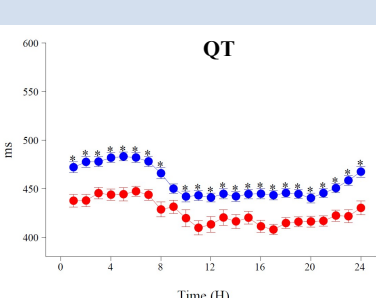
LQT1
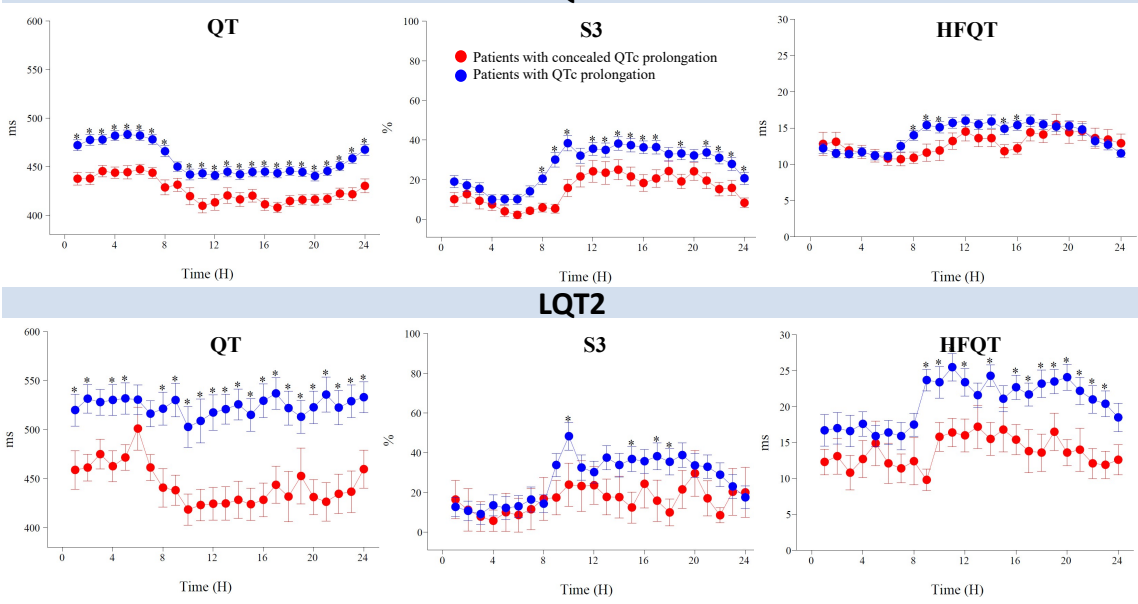

Patients with concealed QTc Prolongation vs Healthy subjects

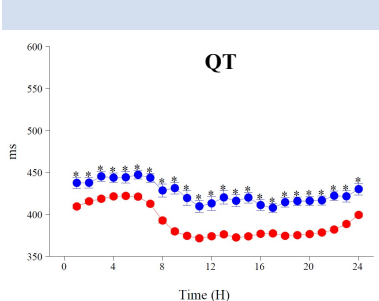
LQT1
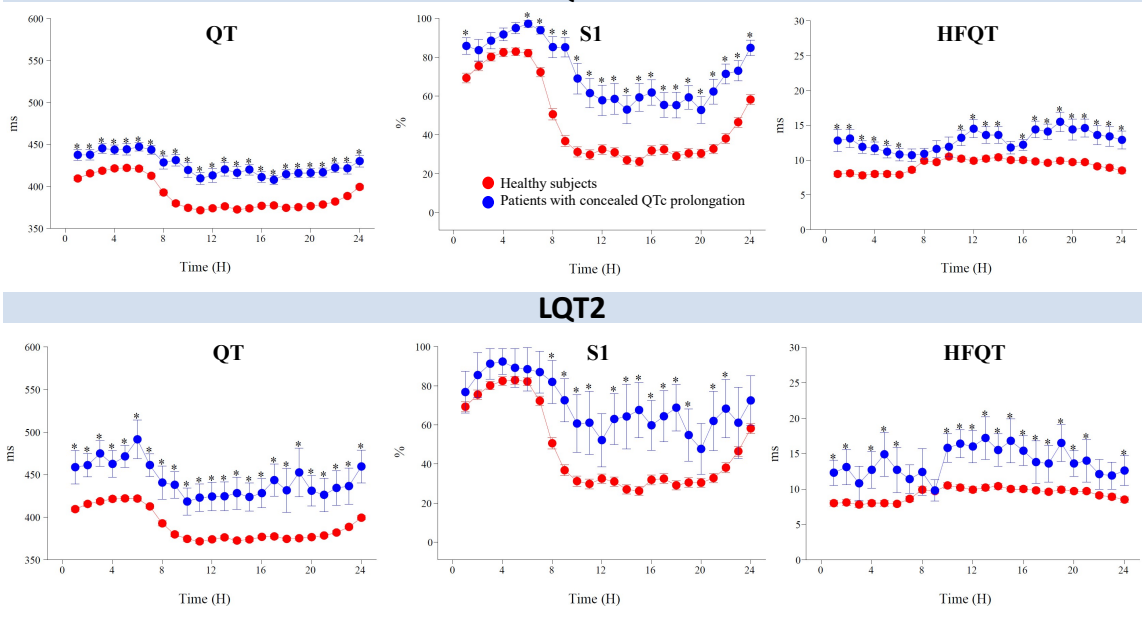


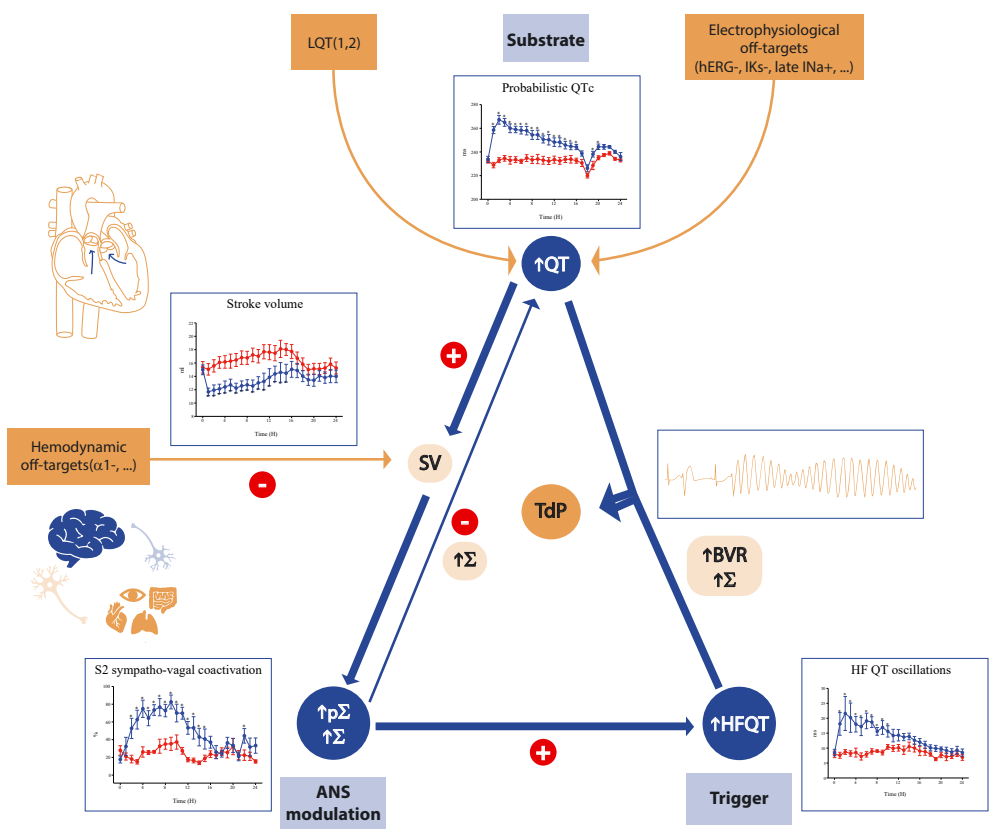

\section{Hosted file}

table_1.pptx available at https://authorea.com/users/396941/articles/510018-reflex-sympathovagal-coactivation-and-concealed-qtc-prolongation-lessons-from-herg-blockers-and-longqt-syndromes-type-1-and-type-2

\section{Hosted file}

table_2.pptx available at https://authorea.com/users/396941/articles/510018-reflex-sympathovagal-coactivation-and-concealed-qtc-prolongation-lessons-from-herg-blockers-and-longqt-syndromes-type-1-and-type-2 